Mini Review

Copyright (C) All rights are reserved by Clement Boateng Ampadu

\title{
A New T-X Family of Distributions
}

\section{Clement Boateng Ampadu*}

Department of Biostatistics, USA

*Corresponding author: Clement Boateng Ampadu, Department of Biostatistics, USA.

Received Date: June 26, 2019

Published Date: August 26, 2019

\section{Abstract}

This paper introduces a new weight in the T - X (W) framework, and shows a new family induced by this new weight is good in fitting real-life data.

Keywords: Exponential distribution; Normal distribution; T-X family of distributions

\section{The T - X(W) Family of Distributions}

This family of distributions is a generalization of the the betagenerated family of dis-tributions first proposed in [1] In particular, let $r(t)$ be the PDF of the random variable $\mathrm{T} \in[\mathrm{a}, \mathrm{b}],-\infty \leq \mathrm{a}<\mathrm{b}$ $\leq \infty$, and let $\mathrm{W}(\mathrm{F}(\mathrm{x})$ ) be a monotonic and absolutely continu-ous function of the CDF F (x) of any random variable X. The CDF of a new family of distributions defined in [2] is given by

$$
G(x)=\int_{a}^{W(F(x))} r(t) d t=R\{W(F(x))\}
$$

where $\mathrm{R}(\cdot)$ is the $\mathrm{CDF}$ of the random variable $\mathrm{T}$ and $\mathrm{a} \geq 0$

Remark 3.1: The PDF of the T - X(W) family of distributions is obtained by differentiating the CDF above

Remark 3.2. When we set $\mathrm{W}(\mathrm{x}):=-\log (1-\mathrm{x})$, then we use the term "T-X" to de-scribe all sub-classes of the T-X(W) family of distributions induced by the weight function $\mathrm{W}(\mathrm{x})=-\log (1-\mathrm{x})$. A description of different weight functions that are appropriate given the support of the random variable $\mathrm{T}$ is discussed in [2]

A plethora of results studying properties and application of the $\mathrm{T}-\mathrm{X}(\mathrm{W})$ family of distri-butions have appeared in the literature, and the research papers, assuming open access, can be easily obtained on the web via common search engines, like Google, etc

The $\left(\frac{1}{e}\right)^{\alpha}$ PT-G Family of Distributions

Definition 4.1: [3] Let $\alpha \neq \frac{1}{e}, \alpha>\frac{1}{e}$ and $\xi>0$ where $\alpha$ is the rate scale parameter and $\xi$ is a vector of parameters in the baseline distribution all of whose entries are positive. A random variable $\mathrm{Z}$ is said to follow the $\left(\frac{1}{e}\right)^{\alpha}$ power transform family of power transform family of distributions if the Cumulative Distribution Function (CDF) is given by

$$
F(x ; \alpha, \xi)=\frac{1-e^{-\alpha G(x ; \xi)}}{1-e^{-\alpha}} \quad x \in \mathbb{R},
$$

where the baseline distribution has $\operatorname{CDF} \mathrm{G}(\mathrm{x} ; \xi)$. Proposition 4.2: [3] The PDF of the $\left(\frac{1}{e}\right)^{\alpha}$ power transform
family distribution is given by

$$
f(x ; \alpha, \xi)=\frac{1}{1-e^{-\alpha}}\left(\alpha g(x, \xi) e^{-\alpha G(x ; \xi)}\right)
$$

Where $\alpha \in \mathbb{R}$ and $\alpha \neq 0$ and $x \in[0,1]$ and $\mathrm{G}(\mathrm{x} ; \xi)$ is the baseline cumulative distribution with probability density function $\mathrm{g}(\mathrm{x} ; \xi)$.

\section{The New Weight and the Distribution}

By modifying the parameter space for $\alpha$ in the $\left(\frac{1}{e}\right)^{\alpha}$ PT-Standard Uniform family and using it in Remark 1.2, we define the new weight as

$$
W^{*}(x ; \alpha)=-\log \left(\frac{1-e^{\alpha-a x}}{1-e^{-\alpha}}\right)
$$

where $\alpha \in \mathbb{R}$ and $\alpha \neq 0$ and $x \in[0,1]$. Using this we introduce the following

Definition 5.1: Let $X$ be a random variable with CDF F $(\mathrm{x} ; \xi)$ and PDF $\mathrm{f}(\mathrm{x} ; \xi)$; and let $\mathrm{T}$ be a random variable with support $[0, \infty)$ whose PDF is $\mathrm{r}(\mathrm{t} ; \gamma)$, and whose CDF is R(t; $\gamma)$; let $\xi$ and $\gamma$ be vector of parameters in the distribution of the random variables $\mathrm{X}$ and $\mathrm{T}$, 
respectively. The new $\mathrm{T}-\mathrm{X}$ family distributions has CDF defined by the following

$G(x ; \xi, \gamma, \alpha)=\int_{0}^{-\log \left(\frac{1-e^{\alpha-\alpha F(x ; \xi)}}{1-e^{\alpha}}\right)} r(t ; \gamma)=R\left[-\log \left(\frac{1-e^{\alpha-\alpha F(x ; \xi)}}{1-e^{\alpha}}\right) ; \gamma\right]$

where, $\alpha, x \in \mathbb{R}$, and $\alpha \neq 0$

Remark 5.2: The PDF of the new T-X family of distributions can be obtained by differentiating the CDF above

\section{Practical Illustration}

Assume $\mathrm{X}$ is a normal random variable with $\mathrm{CDF}$

$$
\mathrm{F}(x ; c, d)=\frac{1}{2} \operatorname{erfc}\left(\frac{c-x}{\sqrt{2} d}\right)
$$

Where $c \in \mathbb{R}, d>0$ and $\operatorname{erfc}(z)=1-\frac{2}{\sqrt{\pi}} \int_{0}^{z} e^{-t^{2}} d t$. Also assume the random variable $\mathrm{T}$ is an exponential random variable with $\mathrm{CDF}$

$$
R(t ; b)=1-e^{-b t}
$$

where $t, b>0$. Now the following is immediate from Definition 3.1

Proposition 6.1: The CDF of the new Exponential-Normal family of distributions is given by

$$
G(x ; c, d, b, \alpha)=1-\left(\frac{e^{\frac{1}{2}} \alpha\left(\operatorname{erfc}\left(\frac{c-x}{\sqrt{2} d}\right)+1\right)-1}{e^{\alpha}-1}\right)^{b}
$$

where $c, \alpha \in \mathbb{R}, b, d>0, \alpha \neq 0$, and $\operatorname{erfc}(z)=1-\operatorname{erf}(z)=1-\frac{2}{\sqrt{\pi}} \int_{0}^{z} e^{-t^{2}} d t$.

Remark 6.2: If a random variable $M$ has $\operatorname{CDF}$ given by the previous Proposition we write $M \sim N$ EN (c, d, b, $\alpha$ ) (Figure $1 \& 2$ ) [4]

The fit in Figure 1 and the approximately straight line in Figure 2 indicates the NEN distribution is a good fit.

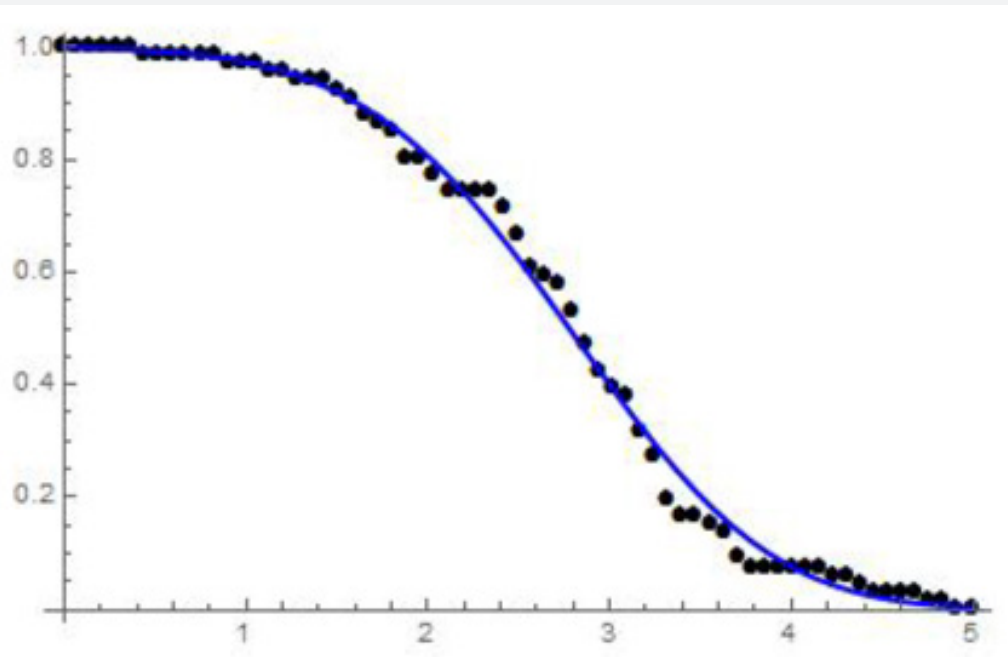

Figure 1: The survival function of NEN(3.11779, 1.0106, 1.62634,-0.184163) fitted to the empirical (survival) distribution.

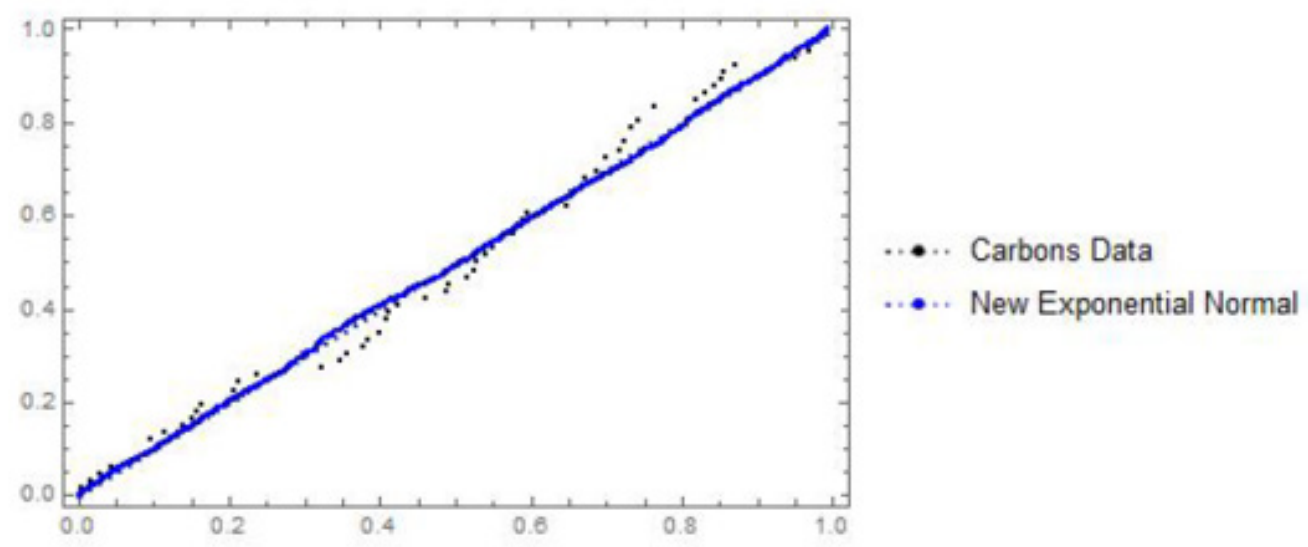

Figure 2: The probability plots of 1500 random samples of $\operatorname{NEN}(3.11779,1.0106,1.62634,-0.184163)$.

By differentiating the CDF, we have the following

Proposition 6.3: The PDF of the new Exponential-Normal family of distributions is given by where

$c, \alpha \in \mathbb{R}, b, d>0, \alpha \neq 0$, and $\operatorname{erfc}(z)=1-\operatorname{erf}(z)=1-\frac{2}{\sqrt{\pi}} \int_{0}^{z} e^{-t^{2}} d t$.

(Figure $3 \& 4$ ) 


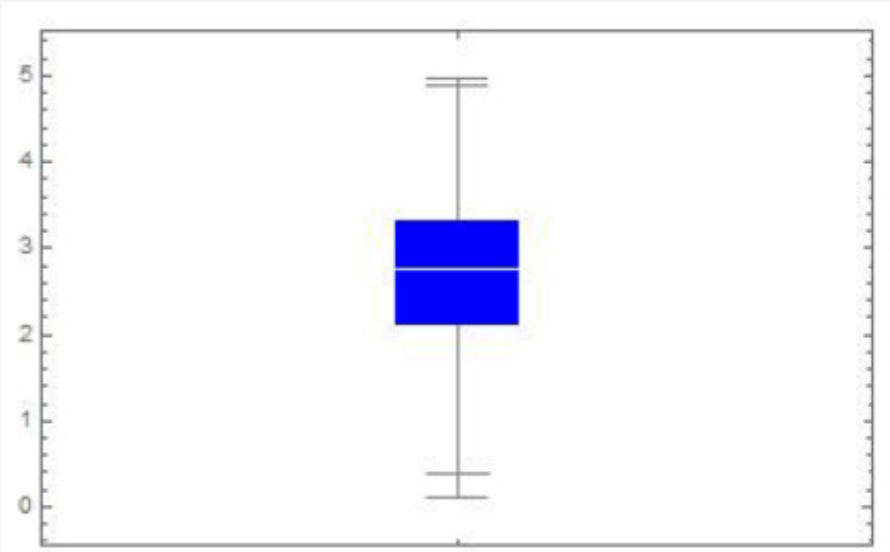

Carbons Data

New Exponential Normal

Figure 3: The box-whisker charts of 1500 random samples of NEN(3.11779, 1.0106, 1.62634,-0.184163).

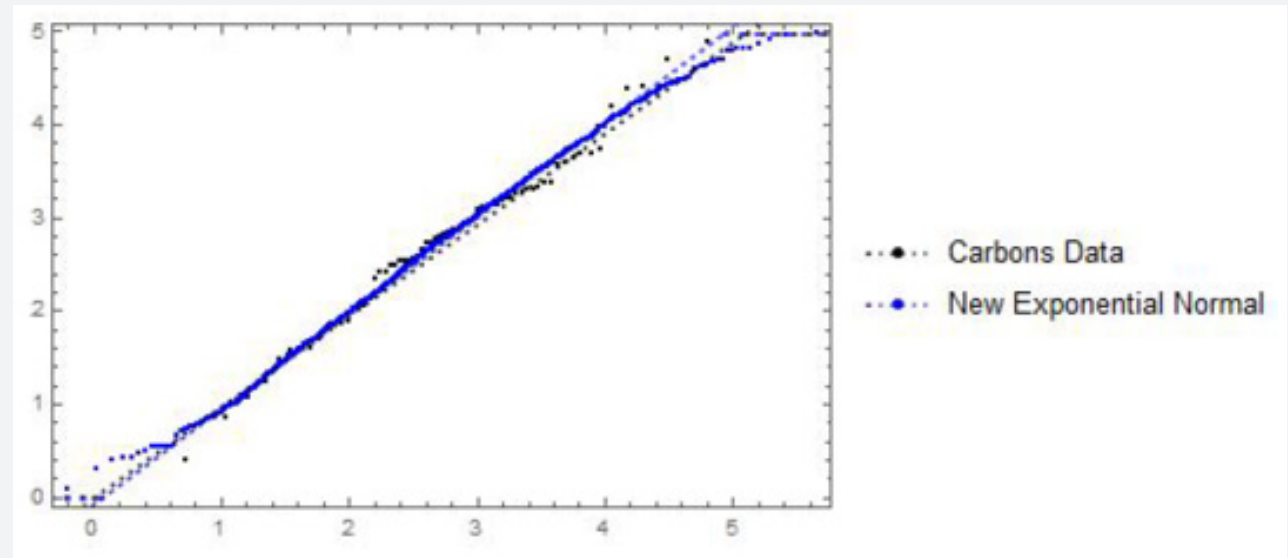

Figure 4: The quantile plots of 1500 random samples of $\mathrm{NEN}(3.11779,1.0106,1.62634,-0.184163)$.

As the box-whisker charts in Figure 3 and the quantile plots in Figure 4 appear almost identical, we have more evidence that the NEN distribution is a good fit.

\section{Concluding Remarks}

This paper has introduced a new T-X family of distributions via Definition 3.1 and has shown a member of this new class of distribtutions is a good fit to real life data. Our hope is that the researchers of distribution theory and its applications will further develop the properties and applications of the new T-X class of distributions.

\section{Acknowledgement}

None.

\section{Conflict of Interest}

No conflict of interest.

\section{References}

1. Eugene N, Lee C, Famoye F (2002) The beta-normal distribution and its applications. Communications in Statistics-Theory and Methods 31(4): 497-512.

2. Alzaatreh A, Lee C, Famoye F (2013b) A new method for generating families of continuous distributions. Metron 71(1): 63-79.

3. Abdulzeid Yen Anafo (2019) The New Alpha Power Transform: Properties and Ap-plications, Master of Science in Mathematical Sciences Essay, African Institute for Mathematical Sciences (Ghana). Unpublished Manuscript.

4. Ayman Alzaatreh, Carl Lee Felix Famoye (2014) T-normal family of distributions: a new approach to generalize the normal distribution, Journal of Statistical Distributions and Applications 1:16. 